\title{
Medians of Discrete Sets according to a Linear Distance
}

\author{
A. Daurat, ${ }^{1}$ A. Del Lungo, ${ }^{2}$ and M. Nivat ${ }^{3}$ \\ ${ }^{1}$ Laboratoire de Logique et d'Informatique de Clermont-1 (LLAIC1), \\ I.U.T. Informatique, Ensemble Universitaire des Cézeaux, \\ B.P. No. 86, 63172 Aubière Cedex, France \\ daurat@1laic.u-clermont1.fr \\ ${ }^{2}$ Dipartimento di Matematica, Università di Siena, \\ Via del Capitano 15, 53100 Siena, Italy \\ dellungo@unisi.it \\ ${ }^{3}$ Laboratoire d'Informatique, Algorithmique, Fondements et Applications (LIAFA), \\ Université Denis Diderot, \\ 2 place Jussieu, 75251 Paris Cedex 05, France \\ Maurice.Nivat@liafa.jussieu.fr
}

\begin{abstract}
In this paper we present some results concerning the median points of a discrete set according to a distance defined by means of two directions $p$ and $q$. We describe a local characterization of the median points and show how these points can be determined from the projections of the discrete set along directions $p$ and $q$. We prove that the discrete sets having some connectivity properties have at most four median points according to a linear distance, and if there are four median points they form a parallelogram. Finally, we show that the 4-connected sets which are convex along the diagonal directions contain their median points along these directions.
\end{abstract}

\section{Introduction}

A discrete set is a nonempty finite subset of the integer lattice $\mathbb{Z}^{2}$. Let $d$ be a distance on $\mathbb{Z}^{2}$. If $\mathcal{S}$ and $P$ are a discrete set and a point $P$ of $\mathbb{Z}^{2}$, we define

$$
D(P)=\sum_{Q \in \mathcal{S}} d(P, Q) .
$$

A point $M$ of $\mathbb{Z}^{2}$ is said to be a median point of $\mathcal{S}$ according to $d$, if

$$
D(M)=\min _{Q \in \mathbb{Z}^{2}} D(Q) .
$$




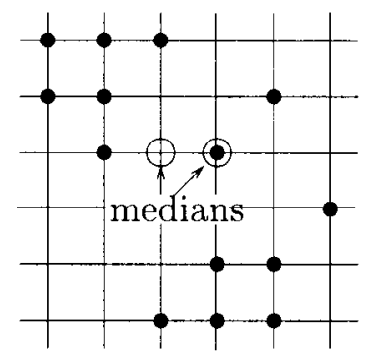

Fig. 1. The median points of a discrete set according to Manhattan distance.

In [7] the authors gave a simple method for determining the medians of a discrete set according to Manhattan distance $d_{1}$ (i.e., $d_{1}(P, Q)=\left|x_{P}-x_{Q}\right|+\left|y_{P}-y_{Q}\right|$ for each $P=\left(x_{P}, y_{P}\right)$ and $Q=\left(x_{Q}, y_{Q}\right)$ belonging to $\left.\mathbb{Z}^{2}\right)$. The discrete set in Fig. 1 has two median points according to $d_{1}$. They showed how the median points can be determined by means of a discrete set's projections along the horizontal and vertical directions. The projection of a discrete set $\mathcal{S}$ along direction $p(M)=a x_{M}+b y_{M}=$ const is the function rp: $\mathbb{Z} \rightarrow \mathbb{N}$ defined by

$$
r p_{i}=\operatorname{card}(\{N \in \mathcal{S} \mid p(N)=i\}) .
$$

This, in turn, means that a projection of a discrete set $\mathcal{S}$ in a direction $p$ is a function giving the number of points of $\mathcal{S}$ on each line parallel to this direction. For instance, Fig. 2 illustrates the projections of a discrete set along the directions $p(M)=x_{M}$ and $q(M)=y_{M}$. The projections along $p(M)=x_{M}, q(M)=y_{M}$ are said to be vertical and horizontal projections, respectively.

The purpose of this paper is to extend the results on the median points given in [7] to the "linear" distances, that is a distance defined in the following way:

$$
d(M, N)=\alpha|p(\overrightarrow{M N})|+\beta|q(\overrightarrow{M N})|
$$

where $\alpha$ and $\beta$ are two positive integer numbers, and $p$ and $q$ are two independent linear

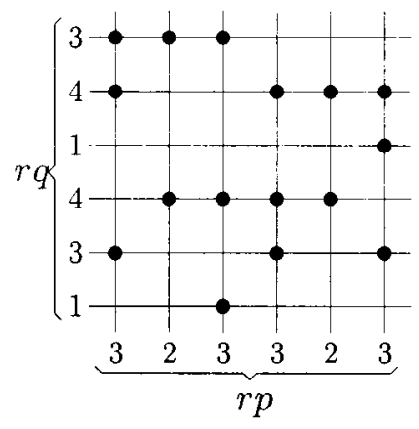

Fig. 2. The projections of a discrete set along the horizontal and vertical directions. 
forms on $\mathbb{Q}^{2}$. We can assume that

$$
\begin{aligned}
& p(\vec{u})=a u_{x}+b u_{y}, \\
& q(\vec{u})=c u_{x}+d u_{y},
\end{aligned}
$$

with $a, b, c, d \in \mathbb{Z}, a d-b c \neq 0, \operatorname{gcd}(a, b)=1$, and $\operatorname{gcd}(c, d)=1$. Note that if $a=1$, $b=0, c=0, d=1$, and $\alpha=\beta=1$, then $d$ is the Manhattan distance.

A priori if we want to verify if a point $M$ is a median point of a discrete $\mathcal{S}$ according to a linear distance we have to compute the function $D$ for all the points of $\mathbb{Z}^{2}$. In Section 3 we show that it is sufficient to compute the function $D$ for only a few points around $M$. From this result follows a simple method to find all the medians of $\mathcal{S}$ by means of its projections along directions $p$ and $q$. In Section 4 we prove that the discrete sets verifying some connectivity properties have at most four median points, and if these sets have four median points they form a parallelogram. In [7] it is proved that a 4-connected set which is convex along the horizontal and vertical directions contains its median points along these directions. Section 5 verifies if this property is true with two other directions. We illustrate that a 4-connected discrete set has this property for the diagonal directions, while there exists a pair of directions for which it is not true.

\section{Some Definitions about Discrete Sets}

Let $\mathcal{S}$ be a discrete set. A column (row) of $\mathcal{S}$ is the intersection of $\mathcal{S}$ with a line $x=k$ $(y=k), k \in \mathbb{Z}$. A north-east diagonal (north-west diagonal) of $\mathcal{S}$ is the intersection of $\mathcal{S}$ with a line $y=x+k(y=-x+k), k \in \mathbb{Z}$.

A 4-neighbor of a point $P=(x, y) \in \mathbb{Z}^{2}$ is a point $P^{\prime}=\left(x^{\prime}, y^{\prime}\right)$ such that $\mid x-$ $x^{\prime}|+| y-y^{\prime} \mid=1$. An 8-neighbor of $P=(x, y)$ is a point $P^{\prime}=\left(x^{\prime}, y^{\prime}\right)$ such that $\left|x-x^{\prime}\right| \leq 1$ and $\left|y-y^{\prime}\right| \leq 1$ (see Fig. 3). Let $P$ and $Q$ be two points of $\mathcal{S}$. An $n$-path from $P$ to $Q$ (with $n=4$ or 8 ) is a sequence of points $P_{0} P_{1} \cdots P_{k}$, where the elements of the sequence belong to $\mathcal{S}, P_{i}$ is an $n$-neighbour of $P_{i-1}$ for $1 \leq i \leq k, P_{0}=P$, and $P_{k}=Q$. A discrete set $\mathcal{S}$ is $n$-connected if there is an $n$-path between any two points $P$ and $Q$ of $\mathcal{S}$ (see Fig. 4).

A discrete set can be represented by a set of cells (unitary squares), as shown in Fig. 4. The cells set corresponding to a 4-connected discrete set is called polyomino. They are well-known combinatorial objects [9], [10] and are related to many different problems, such as tiling [2], [5], enumeration [3], [13], and discrete tomography [1], [8]. In [11] the authors studied the problem of determining the medians of a polyomino, by considering a polyomino as a graph and by using the classical metric on the graph. In

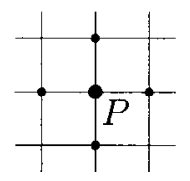

4-neighborhood

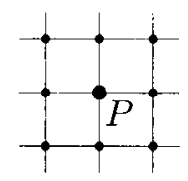

8-neighborhood

Fig. 3. A point $P=(x, y) \in \mathbb{Z}^{2}$ and its neighborhood. 

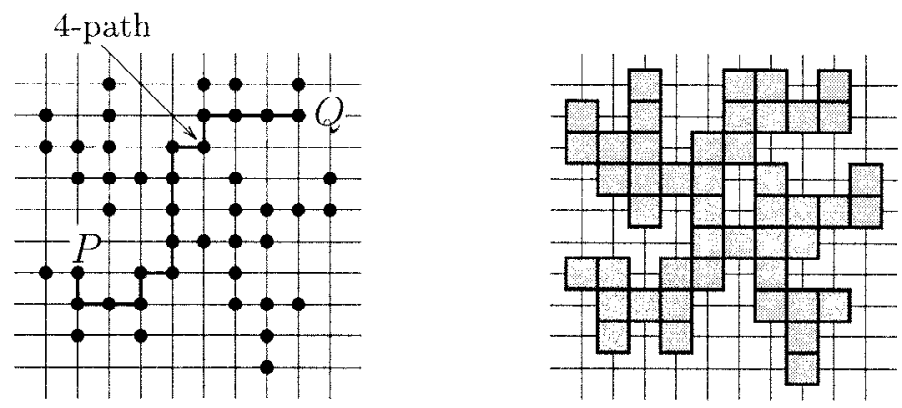

Fig. 4. A 4-connected discrete set and its associated polyomino.

[4] the authors study medians of a rectilinear polygon according to a distance. However, by the definition used in this article the median point is always in the set, but this is not the case for us.

A discrete set is convex if its columns and rows are 4-connected. A discrete set is diagonally convex if its north-east and north-west diagonals are 8-connected. Figure 5 shows two examples of 4-connected discrete sets having the two different kinds of convexity.

\section{Properties of the Medians}

\subsection{Preliminaries}

We denote the line whose equation is $p(M)=i$ by $U_{i}$, and the line whose equation is $q(M)=j$ by $V_{j}$. The intersection of $U_{i}$ and $V_{j}$ is not always in $\mathbb{Z}^{2}$ (see Fig. 6). The following proposition gives a condition to determine if the intersection of these lines is in $\mathbb{Z}^{2}$.

Proposition 3.1. There exists $k$ coprime with $\delta=|a d-b c|$ such that, for all $i, j \in \mathbb{Z}$, $U_{i} \cap V_{j} \in \mathbb{Z}^{2}$ if and only if $j \equiv k i[\delta]$.

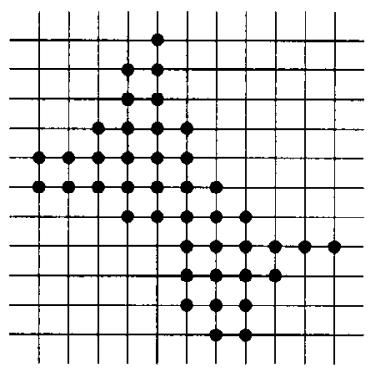

(a)

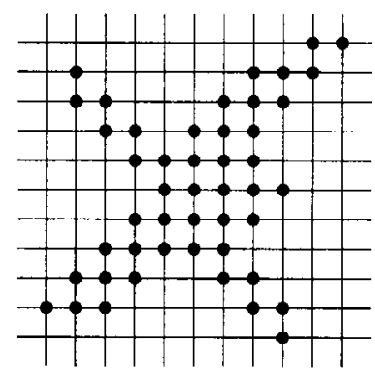

(b)

Fig. 5. (a) A convex set. (b) A diagonally convex set. 


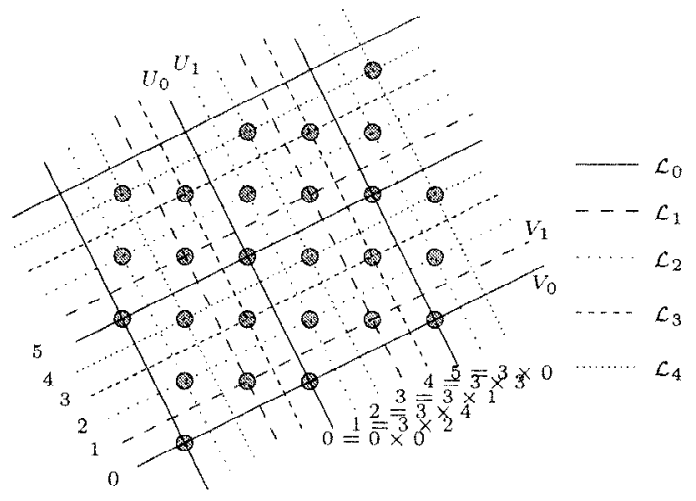

Fig. 6. The integer lattice $\mathbb{Z}^{2}$ and the lines parallel to $p=2 x+y$ and $q=x-2 y$.

Proof. The intersection $U_{i} \cap V_{j} \in \mathbb{Z}^{2}$ if and only if there exists a point $(x, y) \in \mathbb{Z}^{2}$ such that $a x+b y=i$ and $c x+d y=j$; therefore, if and only if there is $(X, Y) \in \mathbb{Z}^{2}$ such that

$$
a(u X-b Y)+b(v X+a Y)=i, \quad c(u X-b Y)+d(v X+a Y)=j .
$$

From the definition of linear distance it follows that $a$ and $b$ are coprime and so there are $u$ and $v$ such that $u a+v b=1$. Consequently, $U_{i} \cap V_{j} \in \mathbb{Z}^{2}$ if and only if there is $(X, Y) \in \mathbb{Z}^{2}$ such that

$$
X=i, \quad(c u+d v) X+(a d-b c) Y=j,
$$

that is, if and only if

$$
\frac{j-(c u+d v) i}{a d-b c} \in \mathbb{Z}
$$

From this condition we deduce that $U_{i} \cap V_{j} \in \mathbb{Z}^{2}$ if and only if $j \equiv k i[\delta]$, where $k=((c u+d v) \operatorname{sign}(a d-b c)) \bmod \delta$.

In the following sections the integers $\delta$ and $k$ denote $|a d-b c|$ and $((c u+d v) \operatorname{sign}(a d-$ $b c)$ ) mod $|a d-b c|$, respectively. Proposition 3.1 brings the following definition:

Definition 1. A subset $\mathcal{L} \subset \mathbb{Z}^{2}$ is a $p$ - $q$-lattice if there is an integer $l \in\{0,1, \ldots \delta-1\}$ such that

$$
\mathcal{L}=\mathcal{L}_{l}=\left\{M \in \mathbb{Z}^{2} \mid q(M) \equiv k p(M) \equiv l[\delta]\right\}
$$

From this definition it follows that $\mathbb{Z}^{2}$ is the union of $\delta$ disjoint $p$ - $q$ lattices. For example, Fig. 6 shows that $\mathbb{Z}^{2}$ is the union of five disjoint $p-q$ lattices: $\mathcal{L}_{0}, \mathcal{L}_{1}, \mathcal{L}_{2}, \mathcal{L}_{3}$, and $\mathcal{L}_{4}$. The interest of this definition is that each $p-q$ lattice $\mathcal{L}_{l}$ is like $\mathbb{Z}^{2}$ with $p=x$ and $q=y$. This, in turn, means that given two points $P$ and $Q$ of $\mathcal{L}_{l}$, the intersection of the line along $p$ and passing through $P$ with the line along $q$ and passing through $Q$ belongs to $\mathcal{L}_{l}$. 


\subsection{Local Characterization}

A priori if we want to verify if a point $M$ is a median point we have to compute the function $D$ for all the points of $\mathbb{Z}^{2}$. In this section we show that it is sufficient to compute the function $D$ for only a few points around $M$.

Definition 2. The neighbor of zero $(\operatorname{denoted} \mathcal{N})$ is the set

$$
\begin{aligned}
\mathcal{N}= & \{\vec{u} \mid \vec{u} \neq \overrightarrow{0},-\delta<p(\vec{u})<\delta,-\delta<q(\vec{u})<\delta\} \\
\cup & \{\vec{u} \mid(p(\vec{u}), q(\vec{u})) \in\{( \pm \delta, 0),(0, \pm \delta)\}\} .
\end{aligned}
$$

We also denote $\mathcal{N}^{\prime}=\{(p(\vec{u}), q(\vec{u})) \mid \vec{u} \in \mathcal{N}\}$. From Proposition 3.1 it follows that

$$
\mathcal{N}^{\prime}=\{(l, m) \in]-\delta, \delta\left[^{2} \mid(l, m) \neq(0,0), m \equiv k l[\delta]\right\} \cup\{( \pm \delta, 0),(0, \pm \delta)\},
$$

and so

$$
\begin{aligned}
\operatorname{card}(\mathcal{N})=\operatorname{card}\left(\mathcal{N}^{\prime}\right) & =4 \operatorname{card}\{(l, m) \in] 0, \delta\left[^{2} \mid m \equiv k l[\delta]\right\}+4 \\
& =4(\delta-1)+4 \\
& =4 \delta
\end{aligned}
$$

For example, $\delta=5$ for the lattice in Fig. 7 and $\operatorname{card}(\mathcal{N})=20$. We point out that $\overrightarrow{0} \notin \mathcal{N}$. For any point $M \in \mathbb{Z}^{2}$ the set

$$
M+\mathcal{N}=\{M+\vec{u} \mid \vec{u} \in \mathcal{N}\}
$$

is called the neighbor of $M$. We now give the main theorem of this section.

Theorem 3.2. A point $M$ is a median of a discrete set $\mathcal{S}$ if and only if,

$$
\forall \vec{u} \in \mathcal{N}, \quad D(M+\vec{u}) \geq D(M) .
$$

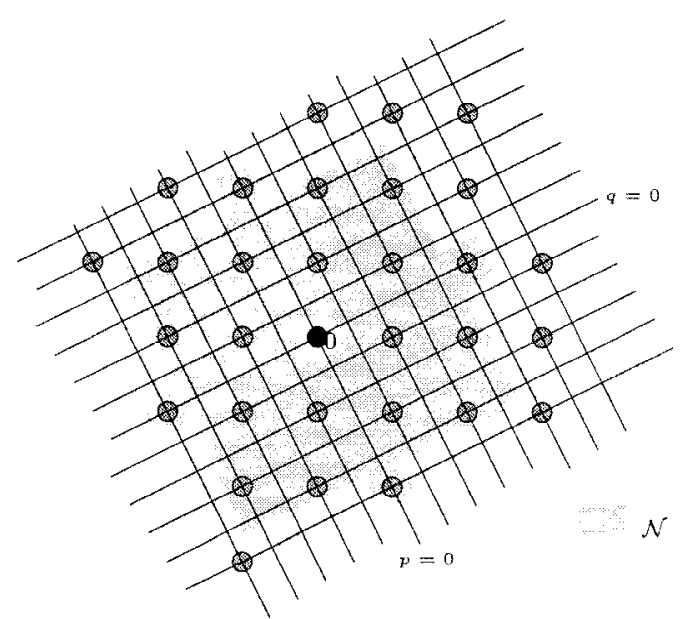

Fig. 7. The neighbor of zero with the directions $p=2 x+y$ and $q=x-2 y$. 
Proof. From the definition of linear distance we deduce that

$$
D(M)=\alpha \sum_{Q \in \mathcal{S}}|p(\overrightarrow{M Q})|+\beta \sum_{Q \in \mathcal{S}}|q(\overrightarrow{M Q})|
$$

By setting

$$
\begin{array}{ll}
D P_{i}=\alpha \sum_{i^{\prime} \in \mathbb{Z}}\left|i-i^{\prime}\right| r p_{i^{\prime}}, & \text { where } \quad r p_{i^{\prime}}=\operatorname{card}\left(\left\{N \in \mathcal{S} \mid p(N)=i^{\prime}\right\}\right), \\
D Q_{j}=\beta \sum_{j^{\prime} \in \mathbb{Z}}\left|j-j^{\prime}\right| r q_{j^{\prime}}, & \text { where } \quad r q_{j^{\prime}}=\operatorname{card}\left(\left\{N \in \mathcal{S} \mid q(N)=j^{\prime}\right\}\right),
\end{array}
$$

it follows that

$$
D(M)=D P_{p(M)}+D Q_{q(M)} .
$$

We prove that the functions $D P$ and $D Q$ are decreasing then increasing functions. Let

$$
\begin{aligned}
S P_{i} & =\sum_{i^{\prime} \leq i} r p_{i^{\prime}}=\operatorname{card}(\{M \in \mathcal{S} \mid p(M) \leq i\}), \\
S P_{i}^{\prime} & =\sum_{i^{\prime} \geq i} r p_{i^{\prime}}=\operatorname{card}(\{M \in \mathcal{S} \mid p(M) \geq i\}), \\
S Q_{j} & =\sum_{j^{\prime} \leq j} r q_{j^{\prime}}=\operatorname{card}(\{M \in \mathcal{S} \mid q(M) \leq j\}), \\
S Q_{j}^{\prime} & =\sum_{j^{\prime} \geq j} r q_{j^{\prime}}=\operatorname{card}(\{M \in \mathcal{S} \mid q(M) \geq j\}) .
\end{aligned}
$$

We have

$$
S P_{i}+S P_{i+1}^{\prime}=S Q_{j}+S Q_{j+1}^{\prime}=\operatorname{card}(\mathcal{S})
$$

and

$$
\begin{aligned}
D P_{i+1}-D P_{i} & =\alpha \sum_{i^{\prime} \in \mathbb{Z}}\left(\left|i+1-i^{\prime}\right|-\left|i-i^{\prime}\right|\right) r p_{i^{\prime}} \\
& =\alpha\left(\sum_{i^{\prime} \leq i}\left(\left(i+1-i^{\prime}\right)-\left(i-i^{\prime}\right)\right) r p_{i^{\prime}}+\sum_{i^{\prime} \geq i+1}\left(\left(i^{\prime}-i-1\right)-\left(i^{\prime}-i\right)\right) r p_{i^{\prime}}\right) \\
& =\alpha\left(\sum_{i^{\prime} \leq i} r p_{i^{\prime}}+\sum_{i^{\prime} \geq i+1}-r p_{i^{\prime}}\right) \\
& =\alpha\left(S P_{i}-S P_{i+1}^{\prime}\right) .
\end{aligned}
$$

So we have

$$
D P_{i+1}-D P_{i}=\alpha\left(S P_{i}-S P_{i+1}^{\prime}\right)=\alpha\left(2 S P_{i}-\operatorname{card}(\mathcal{S})\right) .
$$

In the same way we have

$$
D Q_{j+1}-D Q_{j}=\beta\left(S Q_{j}-S Q_{j+1}^{\prime}\right)=\beta\left(2 S Q_{j}-\operatorname{card}(\mathcal{S})\right)
$$



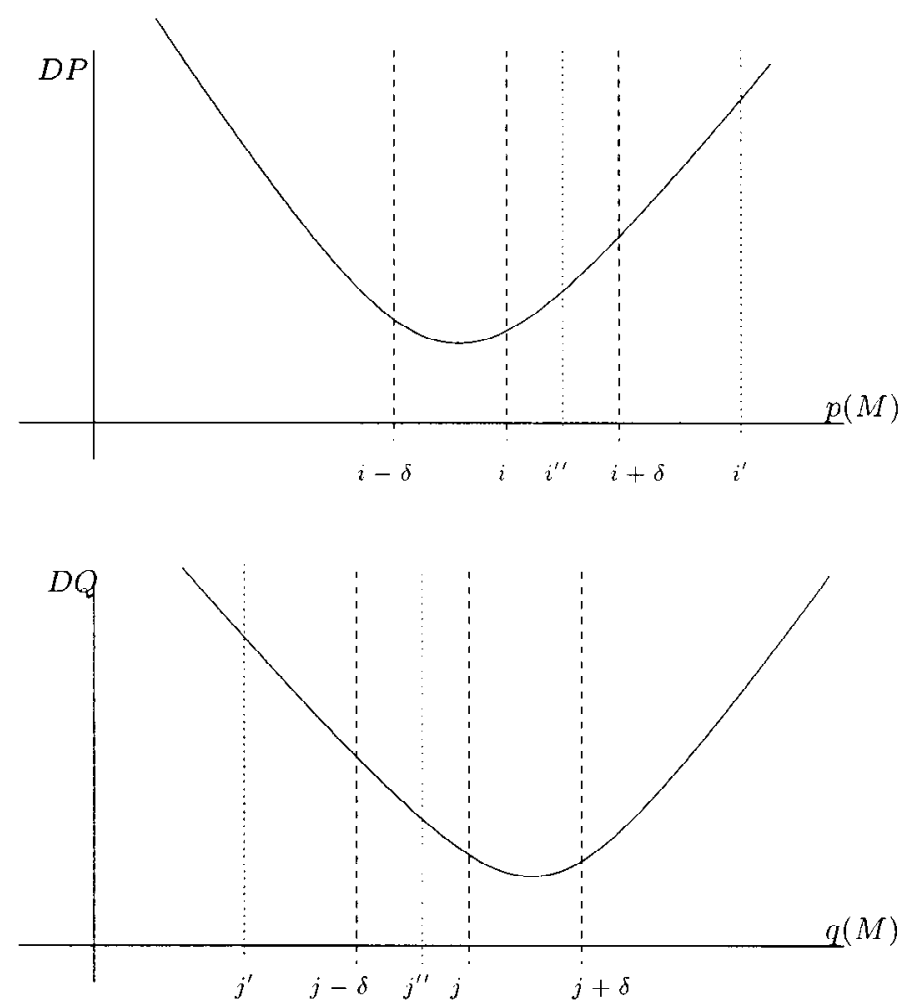

Fig. 8. The functions $D P$ and $D Q$.

Since $\alpha$ and $\beta$ are positive integer numbers, we obtain

$$
\begin{aligned}
D P_{i+1}-2 D P_{i}+D P_{i-1} & =2 \alpha r p_{i} \geq 0, \\
D Q_{j+1}-2 D Q_{j}+D Q_{j-1} & =2 \beta r q_{j} \geq 0,
\end{aligned}
$$

that is, $D P$ and $D Q$ are convex functions (see Fig. 8).

We now prove the theorem. If $M$ is a median point, it obviously verifies condition (3.1). Vice versa, we assume that $M$ verifies (3.1). We have to prove that $M$ is a global minimum of $D$. Let $N$ be a point of $\mathbb{Z}^{2}$. We are going to look for a point $P$ which is in the neighbor of $M$ and which verifies $D(N) \geq D(P)$.

Let $i=p(M), j=q(M), i^{\prime}=p(N)$, and $j^{\prime}=q(N)$. Let $i^{\prime \prime}$ and $j^{\prime \prime}$ be two integer numbers such that

$$
\begin{aligned}
\operatorname{sign}\left(i^{\prime \prime}-i\right)=\operatorname{sign}\left(i^{\prime}-i\right), & \operatorname{sign}\left(j^{\prime \prime}-j\right)=\operatorname{sign}\left(j^{\prime}-j\right), \\
i^{\prime \prime} \equiv i^{\prime}[\delta], & j^{\prime \prime} \equiv j^{\prime}[\delta], \\
-\delta<i^{\prime \prime}-i<\delta, & -\delta<j^{\prime \prime}-j<\delta
\end{aligned}
$$

(see Fig. 8). From Proposition 3.1 it follows that $j^{\prime} \equiv k i^{\prime}[\delta]$. Therefore, $j^{\prime \prime} \equiv k i^{\prime \prime}[\delta]$ and so there is a point $P$ such that $p(P)=i^{\prime \prime}$ and $q(P)=j^{\prime \prime}$. From the definitions of $i^{\prime \prime}$ and $j^{\prime \prime}$ we deduce that $P \in M+\mathcal{N}$ and so $D P_{i^{\prime \prime}}+D Q_{j^{\prime \prime}}=D(P) \geq D(M)=D P_{i}+D Q_{j}$. 
We assume that $i^{\prime} \geq i+\delta$. If $p(\vec{u})=+\delta$ and $q(\vec{u})=0$, then $D(M+\vec{u})=$ $D P_{i+\delta}+D Q_{j}$. Since $(+\delta, 0) \in \mathcal{N}^{\prime}$, from condition (3.1) we have $D(M) \leq D(M+\vec{u})$ and so $D P_{i} \leq D P_{i+\delta}$. The function $D P$ is a decreasing then increasing function and so $D P$ is increasing on $] i+\delta,+\infty$ [. Consequently, $D P_{i} \leq D P_{i+\delta} \leq D P_{i^{\prime}}$. From $\operatorname{sign}\left(i^{\prime \prime}-i\right)=\operatorname{sign}\left(i^{\prime}-i\right),-\delta<i^{\prime \prime}-i<\delta$ and $i^{\prime} \geq i+\delta$, it follows that $i \leq i^{\prime \prime} \leq i^{\prime}$. Since function $D P$ can be an increasing function, or a decreasing then increasing function on $\left[i, i^{\prime}\right]$, we have

$$
D P_{i^{\prime \prime}} \leq \max \left(D P_{i}, D P_{i^{\prime}}\right)=D P_{i^{\prime}} .
$$

If $i^{\prime} \leq i-\delta$, we can prove similarly that $D P_{i^{\prime \prime}} \leq D P_{i^{\prime}}$. Finally, if $\left.i^{\prime} \in\right] i-\delta, i+\delta[$, we get $i^{\prime}=i^{\prime \prime}$, that is $D P_{i^{\prime \prime}}=D P_{i^{\prime}}$. So, we obtain $D P_{i^{\prime}} \geq D P_{i^{\prime \prime}}$ for each position of $i^{\prime}$.

By proceeding analogously we get $D Q_{j^{\prime}} \geq D Q_{j^{\prime \prime}}$. Therefore

$$
D(N)=D P_{i^{\prime}}+D Q_{j^{\prime}} \geq D P_{i^{\prime \prime}}+D Q_{j^{\prime \prime}} \geq D P_{i}+D Q_{j}=D(M)
$$

and so $M$ is a global minimum of $D$.

Remark 1. If we take $p=x$ and $q=y$ and $\alpha=\beta=1$, then the distance $d$ is the Manhattan distance $d_{1}$ which is defined by $d_{1}(M, N)=\left|x_{M}-x_{N}\right|+\left|y_{M}-y_{N}\right|$. In this case, the set $\mathcal{N}^{\prime}=\{( \pm 1,0),(0, \pm 1)\}$ and so the point $M$ with $p(M)=i$ and $q(M)=j$ is a median of $\mathcal{S}$ if and only if

$$
D P_{i} \leq D P_{i-1}, \quad D P_{i} \leq D P_{i+1}, \quad D Q_{j} \leq D Q_{j-1}, \quad D Q_{j} \leq D Q_{j+1},
$$

which can be rewritten by formulas (3.3) and (3.4) as follows:

$$
S P_{i-1} \leq \frac{\operatorname{card}(\mathcal{S})}{2} \leq S P_{i}, \quad S Q_{j-1} \leq \frac{\operatorname{card}(\mathcal{S})}{2} \leq S Q_{j}
$$

The medians according to Manhattan distance have been studied in [7] and its Theorem 3.1 has been found.

Remark 2. If we take the diagonal directions $p=x+y$ and $q=x-y$ and $\alpha=\beta=1$, then the distance $d$ is the distance $2 d_{\infty}$ where $d_{\infty}$ is defined by $d_{\infty}(M, N)=\max \left(\mid x_{M}-\right.$ $\left.x_{N}|,| y_{M}-y_{N} \mid\right)$. The set $\mathcal{N}^{\prime}$ is $\{( \pm 2,0),(0, \pm 2),( \pm 1, \pm 1)\}$ and from formulas (3.3) and (3.4) it follows that a point $M$ is a median for this distance if and only if it verifies the eight inequalities:

$$
\begin{gathered}
S P_{i-2}+S P_{i-1} \leq \operatorname{card}(\mathcal{S}) \leq S P_{i}+S P_{i+1}, \\
S Q_{j-2}+S Q_{j-1} \leq \operatorname{card}(\mathcal{S}) \leq S Q_{j}+S Q_{j+1}, \\
S P_{i}+S Q_{j} \geq \operatorname{card}(\mathcal{S}), \quad S P_{i}+S Q_{j}^{\prime} \geq \operatorname{card}(\mathcal{S}), \\
S P_{i}^{\prime}+S Q_{j} \geq \operatorname{card}(\mathcal{S}), \quad S P_{i}^{\prime}+S Q_{j}^{\prime} \geq \operatorname{card}(\mathcal{S}) .
\end{gathered}
$$

Theorem 3.2 gives a very fast algorithm to decide if a given point is a median point of $\mathcal{S}$. Moreover, we deduce a simple method to find all the medians of $\mathcal{S}$. From formulas (3.3) and (3.4) we deduce that condition (3.1) is equivalent to $4 \delta$ inequalities whose terms are the projections of $\mathcal{S}$ along directions $p$ and $q$. For instance, if $p=x+y$ and $q=x-y$ 


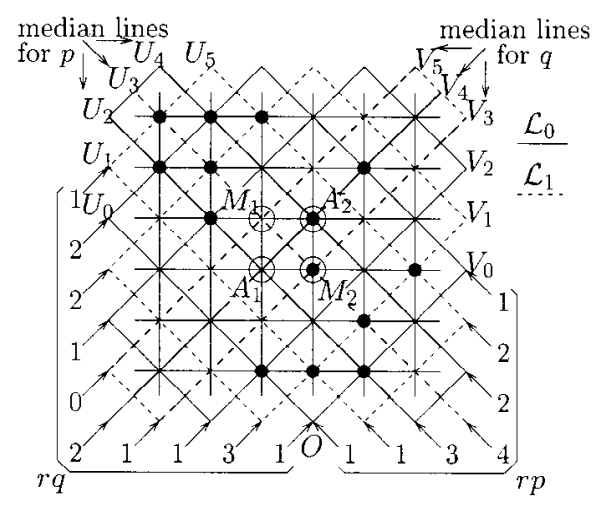

Fig. 9. The median points of a discrete set according to $d_{\infty}$ distance.

and $\alpha=\beta=1$, then condition (3.1) is equivalent to eight inequalities (3.7) whose terms are $S P_{i}$ and $S Q_{j}$ (i.e., the partial sums of the projections of $\mathcal{S}$ along the diagonal directions). Therefore, if we want to determine all the median points of $\mathcal{S}$, we only have to determine the projections $r p$ and $r q$ of $\mathcal{S}$, evaluate the partial sums $S P_{i}$ and $S Q_{j}$ of these projections vectors, and determine the lines satisfying the inequalities on $S P_{i}$ and $S Q_{j}$ equivalent to condition (3.1).

Example 1. We take the discrete set in Fig. 9 and the diagonal directions $p=x+y$ and $q=x-y$ into consideration. We have

$$
\begin{aligned}
r p & =(1,1,3,4,2,2,1) \\
r q & =(1,3,1,1,2,0,1,2,2,1) \\
S P & =(1,2,5,9,11,13,14) \\
S Q & =(1,4,5,6,8,8,9,11,13,14)
\end{aligned}
$$

Moreover, $\operatorname{card}(\mathcal{S})=14, S P_{i}^{\prime}=14-S P_{i-1}$, and $S Q_{j}^{\prime}=14-S Q_{j-1}$. There are only two points satisfying the inequalities (3.7): $M_{1}$ with $p\left(M_{1}\right)=3$ and $q\left(M_{1}\right)=3$,

$$
\begin{gathered}
7=S P_{1}+S P_{2} \leq 14 \leq S P_{3}+S P_{4}=20, \\
9=S Q_{1}+S Q_{2} \leq 14 \leq S Q_{3}+S Q_{4}=14, \\
15=S P_{3}+S Q_{3} \geq 14, \quad 18=S P_{3}+S Q_{3}^{\prime} \geq 14, \\
15=S P_{3}^{\prime}+S Q_{3} \geq 14, \quad 18=S P_{3}^{\prime}+S Q_{3}^{\prime} \geq 14,
\end{gathered}
$$

and $M_{2}$ with $p\left(M_{2}\right)=3$ and $q\left(M_{2}\right)=5$,

$$
\begin{gathered}
7=S P_{1}+S P_{2} \leq 14 \leq S P_{3}+S P_{4}=20, \\
14=S Q_{3}+S Q_{4} \leq 14 \leq S Q_{5}+S Q_{6}=17, \\
17=S P_{3}+S Q_{5} \geq 14, \quad 15=S P_{3}+S Q_{5}^{\prime} \geq 14, \\
17=S P_{3}^{\prime}+S Q_{5} \geq 14, \quad 15=S P_{3}^{\prime}+S Q_{5}^{\prime} \geq 14 .
\end{gathered}
$$

Therefore, the medians points of this set according to $d_{\infty}$ are $M_{1}$ and $M_{2}$. 
In [7] the authors introduce the concept of median rows and columns of a discrete set $\mathcal{S}$, and show that the median points of $\mathcal{S}$ according to Manhattan distance are the intersection of median rows and columns (see Theorem 3.1 in [7]). We can generalize this concept:

— the line $U_{i}$, whose equation is $p(M)=i$, is a median line for $p$ of $\mathcal{S}$ if $D P_{i \pm \delta} \geq$ $D P_{i}$,

- the line $V_{j}$, whose equation is $q(M)=j$, is a median line for $q$ of $\mathcal{S}$ if $D Q_{j \pm \delta} \geq$ $D Q_{j}$.

If a point $M$ is the intersection of a median line for $p$ with a median line for $q$, then

$$
D P_{p(M) \pm \delta} \geq D P_{p(M)}, \quad D Q_{q(M) \pm \delta} \geq D Q_{q(M)} .
$$

From Theorem 3.2 we deduce that $M$ is a minimum of the function $D$ on a $p-q$ lattice $\mathcal{L}_{l}$. Since the number of $p-q$ lattices is $\delta$, we have at least $\delta$ of these minima. The median points are the smallest values of these minima points.

If $p=x, q=y$, there is only one $p$ - $q$ lattice and so the median points of $\mathcal{S}$ are the intersections of the median lines for $p$ with the median lines for $q$. We note that the lines $U_{i}$ and $V_{j}$ are median lines for $p$ and $q$ if they verify inequalities (3.6).

If $p=x+y, q=x-y$, and $\alpha=\beta=1$, there are two $p$ - $q$ lattices: $\mathcal{L}_{0}$ and $\mathcal{L}_{1}$. If a point is the intersection of a median line for $p$ with a median line for $q$, then it is a minimum of $D$ on $\mathcal{L}_{0}$ or $\mathcal{L}_{1}$. For instance, the discrete set in Fig. 9 has three median lines for $p: U_{2}, U_{3}$, and $U_{4}$, and three median line for $q: V_{3}, V_{4}$, and $V_{5}$. Therefore:

- the minima of $D$ on $\mathcal{L}_{0}$ are $A_{1}=U_{2} \cap V_{4}$ and $A_{2}=U_{4} \cap V_{4}$,

- the minima of $D$ on $\mathcal{L}_{1}$ are $M_{1}=U_{3} \cap V_{3}$ and $M_{2}=U_{3} \cap V_{5}$.

Since $D\left(A_{1}\right)=D\left(A_{2}\right)=29$ and $D\left(M_{1}\right)=D\left(M_{2}\right)=28$, the median points of this discrete set are $M_{1}$ and $M_{2}$. We note that the lines $U_{i}$ and $V_{j}$ are median lines for $p=x+y$ and $q=x-y$ if they verify the first two conditions of (3.7). By means of the last two conditions of (3.7) we determine which point between $A_{i}$ and $M_{i}$ has the smallest values of $D$.

\section{The Number of Median Points}

In this section we show that the discrete sets verifying some connectivity properties have at most four median points, and if there are four median points they form a parallelogram. Theorem 3.2 ensures that all the medians points are intersections of a median line for $p$ with a median line for $q$. Moreover, this condition is not sufficient for a pair of directions different from $p=x$ and $q=y$ (see the previous example). Therefore, the number of median points is equal to or smaller than the product of the number of median lines along each direction.

We assume that $p=x$ and $q=y$. In this case the median points of a discrete set $\mathcal{S}$ according to this distance are the intersection of the median lines for $p$ with the median lines for $q$. So, the number of median points is exactly the product of the number of a median line along each direction. The line $U_{i}$ is a median line for $p$ if $S P_{i-1} \leq \operatorname{card}(\mathcal{S}) / 2 \leq S P_{i}$. If $\mathcal{S}$ has at least one point in each vertical line, $S P$ is a 


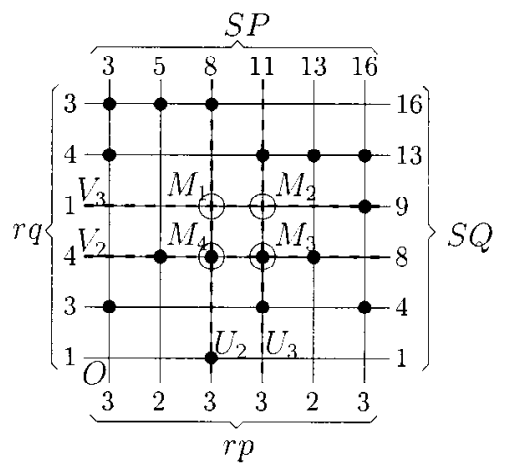

Fig. 10. Median points and lines of a discrete set according to Manhattan distance.

strictly increasing function, and so $\mathcal{S}$ has one or two consecutive median lines for $p$. Consequently, if $\mathcal{S}$ has at least one point in each vertical and horizontal line, then it has at most four medians points.

Example 2. We take the discrete set in Fig. 10 into consideration. It has at least one point in each vertical and horizontal line. Moreover, $r p=(3,2,3,3,2,3)$ and $r q=$ $(1,3,4,1,4,3)$, hence, $\operatorname{card}(\mathcal{S}) / 2=8$, and

$$
\begin{aligned}
& 5=S P_{1} \leq 8 \leq S P_{2}=8 \quad \text { and } \quad S P_{2} \leq 8 \leq S P_{3}=11, \\
& 4=S Q_{1} \leq 8 \leq S Q_{2}=8 \quad \text { and } \quad S Q_{2} \leq 8 \leq S Q_{3}=9,
\end{aligned}
$$

that is, the lines $U_{2}, U_{3}$ and $V_{2}, V_{3}$ verify inequalities (3.6) and so they are median lines for $p$ and $q$. Therefore, there are four median points: $M_{1}=U_{2} \cap V_{3}, M_{2}=U_{3} \cap V_{3}$, $M_{3}=U_{3} \cap V_{2}$, and $M_{4}=U_{2} \cap V_{2}$.

In the general case it is not so simple. Take, for example, $p=x+y$ and $q=x-y$. The line $U_{i}$ is a median line for $p$ if $S P_{i-2}+S P_{i-1} \leq \operatorname{card}(\mathcal{S}) \leq S P_{i}+S P_{i+1}$. If $\mathcal{S}$ has at least one point in each north-west diagonal, $S P$ is a strictly increasing function, and so $\mathcal{S}$ has two or three consecutive median lines for $p$. It follows that if $\mathcal{S}$ has at least one point in each north-east and north-west diagonal, it has at most five medians points (see Fig. 11). We will see that there cannot be five median points because the function $D$ is strictly smaller in the center point than in the four lateral points. We can generalize this result for any direction. At first, we have to define the notion of indivisibility.
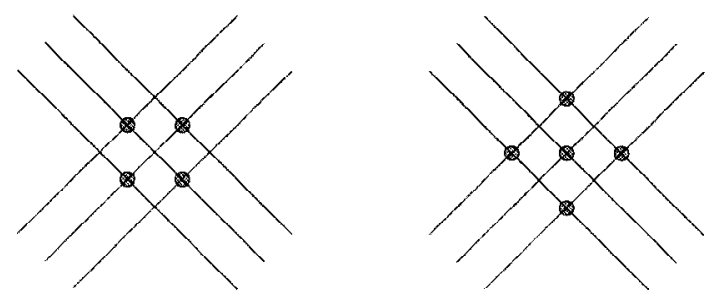

Fig. 11. Intersection of $2 \times 3$ diagonal consecutive lines. 


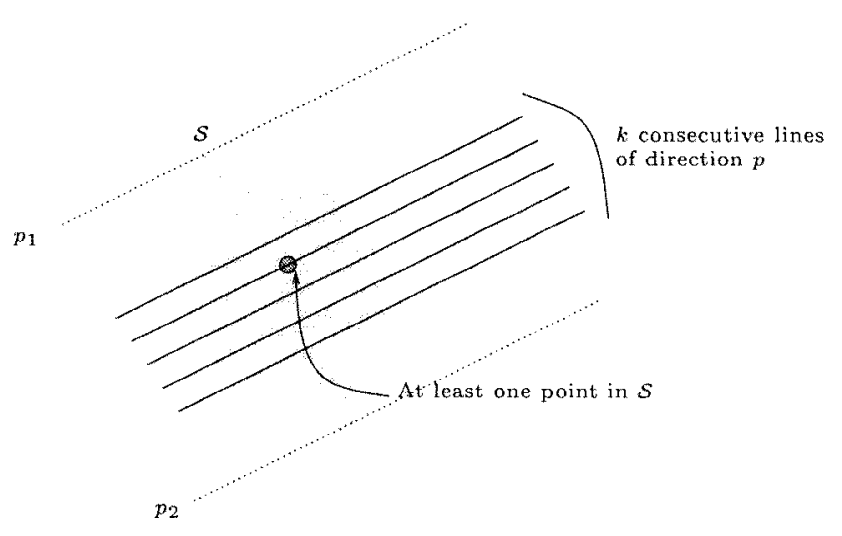

Fig. 12. Definition of $k$ - $p$-indivisibility.

Definition 3. Let $p$ be a linear form and let $k \in \mathbb{N}$. A set $\mathcal{S}$ is $k$ - $p$-indivisible if there are two integers $p_{1}$ and $p_{2}$ so that,

$$
\begin{gathered}
\forall N \in \mathcal{S}, \quad p_{1} \leq p(N) \leq p_{2}, \\
\forall i \in \mathbb{Z}, \quad p_{1} \leq i \leq p_{2}-k+1 \quad \Longrightarrow \quad \exists N \in \mathcal{S}, \quad i \leq p(N) \leq i+k-1
\end{gathered}
$$

(see Fig. 12). A discrete set is 1- $p$-indivisible for $p=x(p=x+y)$ if it has at least one point in each vertical (diagonal) line.

Now, we can show the main result of this section:

Theorem 4.1. If $\mathcal{S}$ is a 1-p-indivisible and 1-q-indivisible discrete set, then $\mathcal{S}$ has at most four median points. Moreover, if $\mathcal{S}$ has exactly four median points, then they form a parallelogram.

We introduce some lemmas for proving this theorem.

Lemma 4.2. Let $f: \mathbb{Z} \rightarrow \mathbb{R}$ be a function which verifies,

$$
\forall x \in \mathbb{Z}, \quad D^{2} f(x)=f(x+1)-2 f(x)+f(x-1) \geq 0,
$$

and let $x_{1}, x_{2}, x_{3}$ be three integers such that $x_{1} \leq x_{2} \leq x_{3}$. If $x=\lambda_{1} x_{1}+\lambda_{2} x_{2}+\lambda_{3} x_{3} \in \mathbb{Z}$, where

$$
\begin{gathered}
\lambda_{1}>0, \quad \lambda_{2} \geq 0, \quad \lambda_{3}>0, \\
\lambda_{1}+\lambda_{2}+\lambda_{3}=1,
\end{gathered}
$$

assuming that there is an integer $\left.x^{\prime} \in\right] x_{1}, x_{3}\left[\right.$ which verifies $D^{2} f\left(x^{\prime}\right)>0$, we obtain

$$
f(x)<\lambda_{1} f\left(x_{1}\right)+\lambda_{2} f\left(x_{2}\right)+\lambda_{3} f\left(x_{3}\right) .
$$


Proof. If $i<j$, we have

$$
\frac{f(j)-f(i)}{j-i}=f(i+1)-f(i)+\sum_{k=i+1}^{j-1} \frac{j-k}{j-i} D^{2} f(k) \geq f(i+1)-f(i),
$$

else, if $j<i$,

$$
\frac{f(i)-f(j)}{i-j}=f(i+1)-f(i)-\sum_{k=j+1}^{i} \frac{k-j}{i-j} D^{2} f(k) \leq f(i+1)-f(i) .
$$

Assuming that $\left.x \in] x_{1}, x_{2}\right],(4.1)$ and (4.2) provide

$$
\begin{aligned}
\frac{f(x)-f\left(x_{1}\right)}{x-x_{1}} & \leq f(x+1)-f(x) \leq \frac{f\left(x_{2}\right)-f(x)}{x_{2}-x}, \\
\frac{f(x)-f\left(x_{1}\right)}{x-x_{1}} & \leq f(x+1)-f(x) \leq \frac{f\left(x_{3}\right)-f(x)}{x_{3}-x} .
\end{aligned}
$$

The existence of $\left.x^{\prime} \in\right] x_{1}, x_{3}\left[\right.$ which verifies $D^{2} f\left(x^{\prime}\right)>0$ gives

$$
\frac{f(x)-f\left(x_{1}\right)}{x-x_{1}}<\frac{f\left(x_{3}\right)-f(x)}{x_{3}-x} \text {. }
$$

and so

$$
\begin{aligned}
& \left(x_{2}-x_{1}\right) f(x) \leq\left(x_{2}-x\right) f\left(x_{1}\right)+\left(x-x_{1}\right) f\left(x_{2}\right), \\
& \left(x_{3}-x_{1}\right) f(x)<\left(x_{3}-x\right) f\left(x_{1}\right)+\left(x-x_{1}\right) f\left(x_{3}\right) .
\end{aligned}
$$

The integer $x=\lambda_{1} x_{1}+\lambda_{2} x_{2}+\lambda_{3} x_{3}$, where $\lambda_{1}, \lambda_{2}$, and $\lambda_{3}$ are three positive or null rational numbers whose sum is 1 . By setting $\alpha=\lambda_{2} / \lambda_{3}$, we obtain

$$
\begin{aligned}
& \lambda_{1}=\frac{1}{\beta}\left(\alpha x_{2}+x_{3}-(1+\alpha) x\right), \\
& \lambda_{2}=\frac{1}{\beta}\left(\alpha\left(x-x_{1}\right)\right), \\
& \lambda_{3}=\frac{1}{\beta}\left(x-x_{1}\right),
\end{aligned}
$$

where $\beta=\alpha x_{2}+x_{3}-(1+\alpha) x_{1}$. By making $\alpha(4.3)+$ (4.4) we have

$$
\begin{aligned}
\left(\alpha x_{2}+\right. & \left.x_{3}-(1+\alpha) x_{1}\right) f(x) \\
& <\left(\alpha x_{2}+x_{3}-(1+\alpha) x\right) f\left(x_{1}\right)+\alpha\left(x-x_{1}\right) f\left(x_{2}\right)+\left(x-x_{1}\right) f\left(x_{3}\right),
\end{aligned}
$$

which is exactly

$$
f(x)<\lambda_{1} f\left(x_{1}\right)+\lambda_{2} f\left(x_{2}\right)+\lambda_{3} f\left(x_{3}\right) .
$$

Remark 3. This lemma is much easier in $\mathbb{R}$ (see for example [12]). Unfortunately, we cannot make the same proof here because the number $\left(\lambda_{1} x_{1}+\lambda_{2} x_{2}\right) /\left(\lambda_{1}+\lambda_{2}\right)$ is not in $\mathbb{Z}$ in general.

From (3.5) it follows that we can apply this lemma to the functions $D P$ and $D Q$, and since $D(M)=D P_{p(M)}+D Q_{q(M)}$, we get: 
Lemma 4.3. Let $\mathcal{S}$ be a 1-p-indivisible and 1-q-indivisible discrete set. Let $A, B$, and $C$ be three points of $\mathbb{Z}^{2}$ which are not all equal. Let $M=\lambda_{A} A+\lambda_{B} B+\lambda_{C} C \in$ $\mathbb{Z}^{2} \backslash\{A, B, C\}$, where $\lambda_{A}, \lambda_{B}$, and $\lambda_{C}$ are three positive rational numbers whose sum is 1. Then

$$
D(M)<\lambda_{A} D(A)+\lambda_{B} D(B)+\lambda_{C} D(C) .
$$

Remark 4. We point out that it is possible to assume that $\mathcal{S}$ is only $(2 \operatorname{det}(p, q)-1)-p$ indivisible and 1- $q$-indivisible in this lemma. In fact, if we do not have $q(A)=q(B)=$ $q(C)$, then $D Q(M)<\lambda_{A} D Q(A)+\lambda_{B} D Q(B)+\lambda_{C} D Q(C)$, that is sufficient to prove the statement of the lemma. If $q(A)=q(B)=q(C), p(A) \leq p(B) \leq p(C)$, and $M \in] A C\left[\cap \mathbb{Z}^{2}\right.$, then we have $p(C)-p(A) \geq 2 \operatorname{det}(p, q)$. So, if $\mathcal{S}$ is $(2 \operatorname{det}(p, q)-1)$ $p$-indivisible, then $D P(M)<\lambda_{A} D P(A)+\lambda_{B} D P(B)+\lambda_{C} D P(C)$.

The following lemma shows that there is no point in the interior of any triangle whose vertices are medians of a discrete set.

Lemma 4.4. Let $\mathcal{E}$ be a subset of $\mathbb{Z}^{2}$ which verifies the following property:

$$
\forall A, B, C \in \mathcal{E}, \quad N \in \operatorname{conv}(A, B, C) \cap \mathbb{Z}^{2} \Longrightarrow N \in\{A, B, C\} .
$$

Then $\mathcal{E}$ has at most four points. Moreover, if $\mathcal{E}$ has four points, then it is a parallelogram.

Proof. Let $A, B, C$, and $M$ be four distinct points of $\mathcal{E}$. Suppose at first that

$$
M \notin \mathcal{I}=\{\alpha A+\beta B+\gamma C \mid \alpha, \beta, \gamma \in \mathbb{Z}, \alpha+\beta+\gamma=1\} .
$$

We have that $\overrightarrow{A M}=u \overrightarrow{A B}+v \overrightarrow{A C}$, with $u$ and $v$ not both integers. We set $M^{\prime}$ so that $\overrightarrow{A M^{\prime}}=u^{\prime} \overrightarrow{A B}+v^{\prime} \overrightarrow{A C}$, with $u^{\prime}=u \bmod 1$ and $v^{\prime}=v \bmod 1$.

If $u^{\prime}+v^{\prime} \leq 1$, then $M^{\prime} \in \operatorname{conv}(A, B, C)$. From the property of $\mathcal{E}$, we deduce that $M^{\prime} \in\{A, B, \bar{C}\}$ and so $M \in \mathcal{I}$. Therefore, we cannot have $u^{\prime}+v^{\prime} \leq 1$. We cannot have $u^{\prime}+v^{\prime} \geq 1$, because in this case $B+\overrightarrow{M^{\prime} C} \in \operatorname{conv}(A, B, C)$ and so $M \in \mathcal{I}$.

Consequently, we must have $M=\alpha A+\beta B+\gamma C$ with $\alpha, \beta, \gamma \in \mathbb{Z}, \alpha+\beta+\gamma=1$. Assume that $A^{\prime}=-A+B+C, B^{\prime}=A-B+C$, and $C^{\prime}=A+B-C$. We are going to prove that $M \in\left\{A^{\prime}, B^{\prime}, C^{\prime}\right\}$. Suppose that it is not the case and take the following different cases into consideration (see Fig. 13):

- If $\alpha \geq 1, \beta \leq 0$, and $\gamma \leq 0$, then $A \in \operatorname{conv}(B, C, M)$ and so $A \in\{B, C, M\}$ for the property of $\mathcal{E}$. Since $A, B, C$, and $M$ are four distinct point of $\mathcal{E}$ this is impossible.

- If $\alpha \leq 0, \beta \geq 1$, and $\gamma \leq 0$, then $B \in \operatorname{conv}(A, C, M)$ and this is impossible.

- If $\alpha \leq 0, \beta \leq 0$, and $\gamma \geq 1$, then $C \in \operatorname{conv}(A, B, M)$ and this is impossible.

- If $\alpha \leq-1, \beta \geq 1$, and $\gamma \geq 1$, then $A^{\prime} \in \operatorname{conv}(B, C, M)$ and this is impossible.

- If $\alpha \geq 1, \beta \leq-1$, and $\gamma \geq 1$, then $B^{\prime} \in \operatorname{conv}(A, C, M)$ and this is impossible.

- If $\alpha \geq 1, \beta \geq 1$, and $\gamma \leq-1$, then $C^{\prime} \in \operatorname{conv}(A, B, M)$ and this is impossible.

Therefore, $M \in\left\{A^{\prime}, B^{\prime}, C^{\prime}\right\}$ and so

$$
\{A, B, C\} \subset \mathcal{E} \subset\left\{A, B, C, A^{\prime}, B^{\prime}, C^{\prime}\right\} .
$$




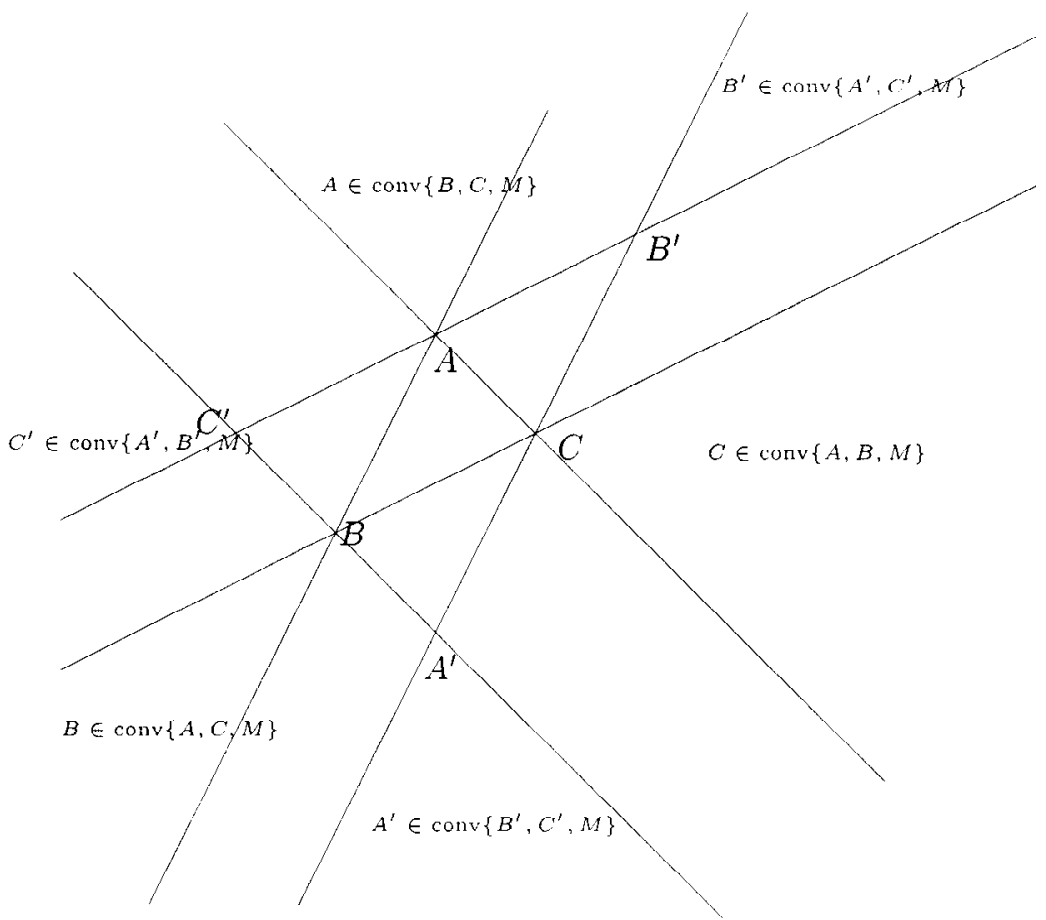

Fig. 13. Where can the point $M$ be put?

We cannot have more than one point in the set $\mathcal{E} \cap\left\{A^{\prime}, B^{\prime}, C^{\prime}\right\}$ because the middle of any two of these points is in $\{A, B, C\}$ and again for the property of $\mathcal{E}$ this is impossible. So, if $\mathcal{E} \neq\{A, B, C\}$, it has got four points and it is a parallelogram.

Finally, we can give the proof of Theorem 4.1.

Proof of Theorem 4.1. Let $\mathcal{E}$ be the set of the median points of $\mathcal{S}$. If we take three distinct points of $A, B, C \in \mathcal{E}$ and a point $M \in \operatorname{conv}(A, B, C)$, then the point $M$ must be in $\{A, B, C\}$. In fact, if $M \notin\{A, B, C\}$, from Lemma 4.3 we have $D(M)<\lambda_{A} D(A)+$ $\lambda_{B} D(B)+\lambda_{C} D(C)$, where $\lambda_{A}, \lambda_{B}$, and $\lambda_{C}$ are three positive rational numbers such that $\lambda_{A}+\lambda_{B}+\lambda_{C}=1$. Since $A, B$, and $C$ are median points, we have $D(A)=D(B)=D(C)$, and so $D(M)<D(A)$. Consequently, $A$ is not a median point. So $M \in\{A, B, C\}$ and we can apply the last lemma which gives the statement of the theorem.

Remark 5. We have proved that a strictly convex function from $\mathbb{Z}^{2}$ to $\mathbb{R}$ reaches its minimum in at most four points.

Remark 6. By Remark 4 and Theorem 4.1, if $\mathcal{S}$ is only $(2 \operatorname{det}(p, q)-1)$ - $p$-indivisible and 1-q-indivisible, then we have also at most four points. 
From the definition of a connected set we obtain:

Property 4.5. Let $\mathcal{S}$ be a discrete set.

- If $\mathcal{S}$ is 8-connected and $p=x$ or $p=y$, then $\mathcal{S}$ is 1-p-indivisible.

- If $\mathcal{S}$ is 4-connected and $p=x+y$ or $p=x-y$, then $\mathcal{S}$ is 1-p-indivisible.

Proof. Consider a point $A \in \mathcal{S}$ which minimizes $p$ and a point $B$ which maximizes $p$. Since $\mathcal{S}$ is $k$-connected ( $k=4$ for the first case and $k=8$ for the second case), there is a $k$-path $\mathcal{C} \subset \mathcal{S}$ which connects the points $A$ and $B$. The $k$-path $\mathcal{C}$ has a common point with all the lines whose direction is $p$ and which are between $A$ and $B$. Therefore, $\mathcal{S}$ is 1- $p$-indivisible.

From Theorem 4.1 and Property 4.5, we obtain the following result:

Corollary 4.6. Let $\mathcal{S}$ be a discrete set.

- If $\mathcal{S}$ is 8-connected, then $\mathcal{S}$ has at most four median points for $d_{1}$.

- If $\mathcal{S}$ is 4-connected, then $\mathcal{S}$ has at most four median points for $d_{\infty}$.

Moreover, if $\mathcal{S}$ has exactly four median points, they form a parallelogram.

\section{Membership of the Diagonal Medians}

In [7] the authors have proved that a 4-connected set which is convex along the horizontal and vertical directions contains its median points along these directions. We have a similar result for the median points according to $d_{\infty}$.

Theorem 5.1. If $\mathcal{S}$ is a 4-connected diagonally convex set, it contains its median points according to the distance $d_{\infty}$

Proof. We assume that $\mathcal{S}$ is a subset of $\mathbb{Z}^{2}$ which satisfies the conditions of the theorem. Let $M$ be a median of $\mathcal{S}$ for the distance $d_{\infty}$. In this case, $p=x+y$ and $q=x-y$ and we define four regions around $M$ (see Fig. 14) as follows:

$$
\begin{aligned}
& R_{0}=\{N / p(N) \leq p(M) \text { and } q(N) \leq q(M)\}, \\
& R_{1}=\{N / p(N) \geq p(M) \text { and } q(N) \leq q(M)\}, \\
& R_{2}=\{N / p(N) \geq p(M) \text { and } q(N) \geq q(M)\}, \\
& R_{3}=\{N / p(N) \leq p(M) \text { and } q(N) \geq q(M)\} .
\end{aligned}
$$

We point out that $M$ belongs to each region. Since $M$ is a median point, we have (3.7):

$$
\begin{array}{ll}
S P_{i}+S Q_{j} \geq \operatorname{card}(\mathcal{S}), & S P_{i}+S Q_{j}^{\prime} \geq \operatorname{card}(\mathcal{S}), \\
S P_{i}^{\prime}+S Q_{j} \geq \operatorname{card}(\mathcal{S}), & S P_{i}^{\prime}+S Q_{j}^{\prime} \geq \operatorname{card}(\mathcal{S}),
\end{array}
$$

where $i=p(M)$ and $j=q(M)$. 


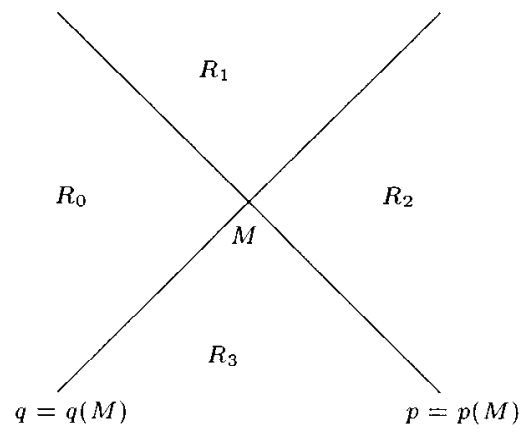

Fig. 14. The four regions.

Suppose that there is no point of $\mathcal{S}$ in $R_{0}$. Then $M \notin \mathcal{S}$ and $S P_{i}+S Q_{j}=\operatorname{card}(\mathcal{S} \cap$ $\left.\left(R_{1} \cup R_{3}\right)\right)$. From the first inequality, it follows that $\operatorname{card}\left(\mathcal{S} \cap\left(R_{1} \cup R_{3}\right)\right) \geq \operatorname{card}(\mathcal{S})$, and so $\mathcal{S} \subset R_{1} \cup R_{3}$ which is impossible because $\mathcal{S}$ is 4 -connected and $M \notin \mathcal{S}$. Therefore the set $\mathcal{S} \cap R_{0}$ is not empty. By proceeding in the same way, we can prove that the set $\mathcal{S} \cap R_{i} \neq \varnothing$ for all $i$.

Let $B_{i}$ be the 4-border of the region $R_{i}$. These borders are unions of two diagonal semilines: $B_{i}=\Delta_{i} \cup \Delta_{i+1}$ (see Fig. 15). Since $\mathcal{S}$ is 4-connected and the sets $\mathcal{S} \cap R_{i}$ are not empty, there is at least a point of $\mathcal{S}$ in each border $B_{i}$. That is, $\mathcal{S} \cap \Delta_{i} \neq \varnothing$ or $\mathcal{S} \cap \Delta_{i+1} \neq \varnothing$.

Consider $\mathcal{S} \cap B_{0}$ and suppose that $\mathcal{S} \cap \Delta_{0} \neq \varnothing$. If $\mathcal{S} \cap \Delta_{2} \neq \varnothing$, then $M \in \mathcal{S}$ follows from diagonal convexity. Conversely, if $\mathcal{S} \cap \Delta_{2}=\varnothing$, since $\mathcal{S} \cap B_{1} \neq \varnothing$ and $B_{1}=\left(\Delta_{1} \cup \Delta_{2}\right)$, we have $\mathcal{S} \cap \Delta_{1} \neq \varnothing$. Moreover, $\mathcal{S} \cap B_{2} \neq \varnothing$ and $B_{2}=\left(\Delta_{2} \cup \Delta_{3}\right)$ and so $\mathcal{S} \cap \Delta_{3} \neq \varnothing$. By the diagonal convexity of $\mathcal{S}, M \in \mathcal{S}$.

Consequently, the median point $M$ is in $\mathcal{S}$.

We could expect that we have a similar membership property for any couple of directions, but unfortunately we cannot simply generalize the previous theorem. For example, if we take $d(M, N)=|p(\overrightarrow{M N})|+|q(\overrightarrow{M N})|$ with $p=x$ and $q=x+4 y$, then the set of Fig. 16 does not contain all its medians along $d$.

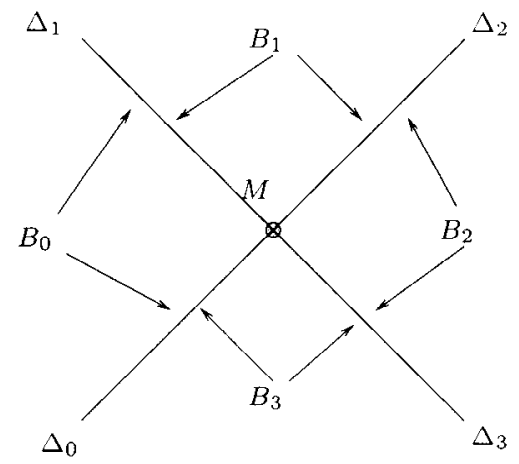

Fig. 15. The four borders. 


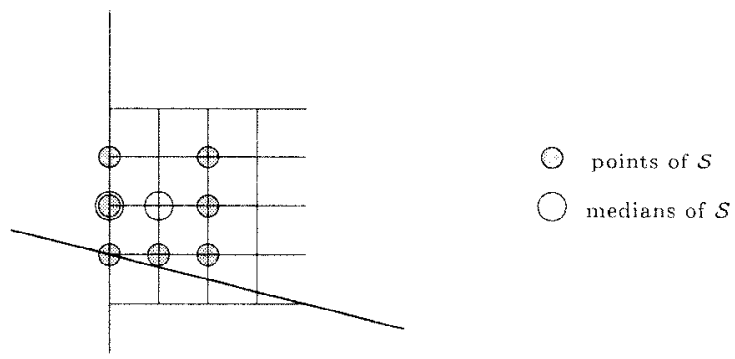

Fig. 16. A set which does not contain its medians.

In fact, if we want to have membership property theorem, we must impose a "stronger" convexity to the sets (see [6]).

\section{Acknowledgements}

The authors thank the anonymous referees for their suggestions.

\section{References}

1. E. Barcucci, A. Del Lungo, M. Nivat, and R. Pinzani, Reconstructing convex polyominoes from their horizontal and vertical projections, Theoret. Comput. Sci. 155 (1996), 321-347.

2. D. Beauquier and M. Nivat, Tiling the plane with one tile, Topology and Category Theory in Computer Science, Oxford University Press, Oxford, 1991, pp. 291-334.

3. M. Bousquet-Mélou, A method for the enumeration of various classes of column-convex polygons, Discrete Math. 154 (1996), 1-25.

4. V. Chepoi and F. Dragan, Computing a median point of a simple rectilinear polygon. Inform. Process Lett. 49 (1994), 281-285.

5. J. H. Conway and J. C. Lagarias, Tiling with polyominoes and combinatorial group theory, J. Combin. Theory Ser. A 53 (1990), 183-208.

6. A. Daurat, The Q-convexity, preprint, 1999.

7. A. Del Lungo, M. Nivat, R. Pinzani, and L. Sorri, The medians of discrete sets, Inform. Process Lett. 65 (1998), 293-299.

8. R. J. Gardner, P. Gritzmann, and D. Prangenberg, On the computational complexity of reconstructing lattice sets from their X-rays, preprint, 1997.

9. S. W. Golomb, Polyominoes, revised and expanded edition, Princeton University Press, Princeton, NJ, 1994.

10. D. A. Klarner, My life among the polyominoes, in The Mathematical Gardner, Wadsworth, Belmont, CA, 1981, pp. 243-262..

11. Y. Métivier and N. Saheb, Medians and centres of polyominoes, Inform. Process Lett. 57 (1996), 175-181.

12. L. Schwartz. Analyse, volume II, Hermann, Paris 1992, Chapter 3.2, p. 52.

13. X. G. Viennot, A Survey of Polyomino Enumeration, Proc. Séries formelles et combinatoires algébrique, Montréal, Juin 1992, Publications du LACIM 11, Université du Québec a Montréal, 1992.

Received April 20, 1999, and in revised form November 16, 1999. 